\title{
QUEEN'S
UNIVERSITY
BELFAST
}

\section{Influence of fill factor variation in high shear granulation on the post granulation processes: Compression and tablet properties}

Mangwandi, C., Adams, M. J., Hounslow, M. J., \& Salman, A. D. (2014). Influence of fill factor variation in high shear granulation on the post granulation processes: Compression and tablet properties. Powder Technology, 263, 135-141. https://doi.org/10.1016/j.powtec.2014.05.005

Published in:

Powder Technology

Document Version:

Early version, also known as pre-print

Queen's University Belfast - Research Portal:

Link to publication record in Queen's University Belfast Research Portal

\section{General rights}

Copyright for the publications made accessible via the Queen's University Belfast Research Portal is retained by the author(s) and / or other copyright owners and it is a condition of accessing these publications that users recognise and abide by the legal requirements associated with these rights.

Take down policy

The Research Portal is Queen's institutional repository that provides access to Queen's research output. Every effort has been made to ensure that content in the Research Portal does not infringe any person's rights, or applicable UK laws. If you discover content in the Research Portal that you believe breaches copyright or violates any law, please contact openaccess@qub.ac.uk. 
1 Influence of fill factor variation in high shear granulation on the post 2 granulation processes: compression and tablet properties.

3

4

5

6

$7 \quad{ }^{2}$ School of Chemistry \& Chemical Engineering, Queen's University Belfast, Belfast BT9 5AG, UK
Corresponding authors

E-mail: Addresses

c.mangwandi@qub.ac.uk (C.Mangwandi),Tel. +44(0)2890974378 


\section{Abstract}

16 This paper describes an investigation of the effect of fill factor; on the compaction behaviour of the

17 granules during tableting and hence mechanical properties of tablets formed. The fill factor; which

18 is the ratio of volume of wet powder material to vessel volume of the granulator, was used as an

19 indicator of batch size. It has been established previously that in high shear granulation the batch

20 size influences the size distribution and granule mechanical properties [1]. The work reported in this

21 paper is an extension to the work presented in [1], hence granules from the same batches were used

22 in production of tablets. The same tabletting conditions were employed during tabletting to allow a

23 comparison of their properties. The compaction properties of the granules are inferred from the data

24 generated during the tabletting process. The tablet strength and dissolution properties of the tablets

25 were also measured. The results obtained show that the granule batch size affects the strength and

26 dissolution of the tablets formed. The tablets produced from large batches were found to be weaker

27 and had a faster dissolution rate. The fill factor was also found to affect the tablet to tablet variation

28 of a non-functional active pharmaceutical ingredient included in the feed powder. Tablets produced

29 from larger batches show greater variation compared to those from smaller batches.

30 Keywords: fill factor, compression, granules strength, compaction energy, batch size 


\section{1. Introduction}

High shear wet granulation has been used extensively in the pharmaceutical industrial as a

35 size enlargement process for granulating feed powders in order to improve their flow characteristics.

36 Moreover, it has been used in a number industries for the manufacture of different products, e.g.

37 fertilisers in agro-based industries [2-4], and for the granulation and mixing of metal or powder

38 oxides such as iron, silica and aluminium in the metal processing industry [5, 6]. The quality of the

39 granules formed during this process is sensitive to the process conditions as well as the formulation

40 [1, 7-11]. Several studies have been undertaken to investigate the importance of process variables on

41 the granule size and size distribution [8, 12-15]. Research on scale-up has focused particularly on

42 the influence of the size distribution of the product [7, 12, 16-20]. Hassapour et al. [21] and

43 Rahmanian et al. [22] examined scale-up rules based on constant speed, shear stress and the Froude

44 number to achieve a target granule strength. It was concluded that a constant tip speed was the most

45 effective. However, even when using the same granulator, small variations in the size of the batch

46 can lead to significant differences in the properties of the granules [10, 12, 20, 23-25].

47 The fill factor is defined as the ratio of the volume of wet powder material to the vessel

48 volume of the granulator. Recent work has shown that not only is the granules size affected by the

49 variations in the fill factor but also the mechanical properties of the granules formed [1]. The total 50 mass of the granulate material was varied (from 2113 to $2875 \mathrm{~g}$ corresponding to fill factors of 0.21

51 to 0.42 respectively) without changing the other variables such as impeller speed, granulation time

52 and liquid to solid ratio. The resulting mechanical properties, such as strength, yield stress and

53 Young's modulus, of the granules were measured. The granule strength, Young's modulus and yield

54 stress of the granules were shown to increase with increasing batch size as represented by the fill 55 factor. 
The implications of batch size variation on the downstream processes due to changes in the

57 material properties have not been investigated and this is the objective of the current work. The

58 main aim was to establish the effects of the fill factor on the compression behaviour of the granules

59 and the consequent effect on the tablet properties. The fill factor was varied by changing the total

60 mass of the feed powder and binder liquid without changing other variables (impeller speed, liquid-

61 to-solid ratio and granulation time) as described in previous work by the current authors [1]. It was

62 found out that changing the fill factor of the granulator resulted in changes in the size distribution

63 and mechanical properties of the granules produced.

64 The behaviour of granular solids under compression depends on the mechanical properties of

65 the granules and this in turn has an effect on the mechanical properties of the tablets formed. A

66 number of parameters that characterise the compression behaviour were determined (efficacy

67 coefficient, net compression work and degree of compression), which will be described in the next

68 section. The objective of this paper was to study the effect of fill factor on the mechanical,

69 dissolution, and homogeneity of tablets formed from high shear granules. The effects of the fill

70 factor on the strength and mean dissolution times of tablets formed from the granules were also

71 measured. Although previous studies have considered the compositional uniformity of tablets [26-

72 30], the effect of granulation process variables on tablet homogeneity has not been addressed.

\section{2. Materials and Methods}

\section{$74 \quad 2.1 \quad$ Production of the granules and tablets}

75 Granules were produced in a $10 \mathrm{~L}$ high shear granulator (RomatoRoto Junior) from a mixture

76 of lactose monohydrate powder (Granulac 230, MolkerelMeggelGmBH, German) and potato starch

77 (Solani, Pharma, Quality Avebe) using an aqueous solution of hydroxypropyl cellulose (HPC) as

78 the binder. Sodium chloride was added to the powder mixture $(1 \% \mathrm{w} / \mathrm{w})$ as non-functional active 
79 ingredient. In all the experiments, the feed powder was pre-mixed at an impeller speed of $250 \mathrm{rpm}$

80 for $2 \mathrm{~min}$. The subsequent inclusion of the binder involved pouring for a period of about $1 \mathrm{~min}$ with

81 an additional granulation period of $6 \mathrm{~min}$ [1]. The granules were dried in a fluidised bed at a

82 temperature of $50^{\circ} \mathrm{C}$ to a moisture content of approximately $4 \% \mathrm{w} / \mathrm{w}$, which required a drying time

83 of about $25 \mathrm{~min}$. The fill factor was calculated from the following expression [1]:

$84 \psi=\frac{m_{w}}{\rho_{w} \pi R_{B}^{2} H}$

Eq (1)

85

86 where $m_{w}$ and $\rho_{w}$ are the mass and bulk density of the wet powder, and $R_{B}$ and $H$ are the radius and

87 height of the cylindrical granulator vessel. The bulk densities of the dried granules, $\rho_{b}$, in the size

88 range 0.5 to $0.6 \mathrm{~mm}$, from the different batches, were determined by measuring the mass, $m$, of a

89 known volume of granules, $V$ :

$90 \quad \rho_{b}=\frac{m}{V}$

Eq (2)

\section{$91 \quad$ 2.1.1 Production of tablets}

$92100 \mathrm{mg}$ tablets were also produced from the granules in the size range $0.5-0.6 \mathrm{~mm}$ at a

93 maximum compression force of $5 \mathrm{kN}$ using a universal material tester (Instron model 3555); the

94 loading and unloading data were stored in a computer. The loading and unloading speeds were both

$9510 \mathrm{~mm} / \mathrm{min}$ and the internal diameter of the die was $6.35 \mathrm{~mm}$. The tablets were stored in sealed

96 plastic bags before their strength and dissolution characteristics were measured. The force -

97 displacement data was recorded during compression of bed of granules into tablet and was used to

98 determine the strength of the granules as described in section 2.1.2.

99 
101

102

103

104 obtain the single granule strength:

$105 \quad \ln P=\ln \left(\frac{\tau}{\alpha}\right)+\alpha \varepsilon_{n}+\ln \left(1-e^{\left(-\alpha \varepsilon_{n}\right)}\right)$

106

113 tablets, $W_{e}$, was calculated from the integral of the unloading force data:

$114 \quad W_{e}=\frac{1}{m_{b}} \int_{\Delta_{m}}^{\Delta_{0}} F_{u n l}(\Delta) \mathrm{d} \Delta$

115 where $F_{u n l}(\Delta)$ is the force during unloading, $m_{b}$ is the mass of the bed of granules in the die; $\Delta_{0}$ and $\Delta_{m}$ correspond to the displacement at zero and maximum loading respectively. The net compaction

117 work, $W_{\text {net }}$, which represents the energy dissipated, corresponds to the difference between the 118 integrals of the loading and unloading curves:

$$
W_{\text {net }}=\frac{1}{m_{b}}\left(\int_{0}^{\Delta_{\max }} F_{l}(\Delta) \mathrm{d} \Delta-W_{e}\right)
$$

where $F_{l}(\Delta)$ is the force during loading. 
123 The degree of compression was determined from the initial bed height, $h_{0}$, and bed height at

124 maximum compression pressure, $h_{\max }$ using [32]:

125

$$
C_{p}=\left(\frac{h_{0}-h_{\max }}{h_{0}}\right) \times 100 \%
$$

127 This parameter corresponds to the maximum percent engineering compressive strain.

\subsubsection{Tablet tensile strength}

The tablets were compressed diametrically at a speed of $2 \mathrm{~mm} / \mathrm{min}$, until fracture occurred and

131 each experimental condition and compact type. The strength of the tablets, $\sigma_{t}$, was calculated from

132 the maximum load, $F_{\max }$ and the dimensions of the tablet, i.e. the tablet diameter $D_{t}$ and thickness, $x$ $133 \quad[33,34]:$

$$
\sigma_{t}=2 \frac{F_{\max }}{\pi x D_{t}}
$$

137 of the force-displacement curve:

$$
W_{t}=\frac{1}{m_{t}} \int_{0}^{\delta_{\max }} F(\delta) \mathrm{d} \delta
$$




\section{$144 \quad$ 2.1.5 Efficacy of compression coefficient}

145 The efficacy of compression coefficient, $C_{\text {eff, }}$ which expresses the ability of the granules to 146 convert the net compression energy into cohesion energy, was determined [35, 36]. The cohesion 147 energy is that required to form bonds between the granules during compression:

$$
C_{\text {eff }}=\frac{W_{t}}{W_{\text {net }}} \times 100 \%
$$

Values $>0.1 \%$ are characteristic of an effective conversion of net compression work into cohesion

151 [36-39]. The strength of the tablets formed during compression is linked to amount of cohesion

152 between the constituents of the tablet; higher cohesion would result in formation of stronger tablets

153 whereas lower cohesion would be linked to formation of weaker tablets. Hence efficacy of 154 compression is of particular interest to this study.

\subsection{Tablet dissolution}

156 The dissolution of $100 \mathrm{mg}$ tablets in $250 \mathrm{ml}$ distilled water was measured at a temperature of $15737^{\circ} \mathrm{C}$. This involved stirring with a paddle at $250 \mathrm{rpm}$ and monitoring the conductivity of the 158 solution as a function of time using a conductivity meter (Hanna 9000, Hanna Instruments, USA). 159 The conductivity and temperature data were recorded automatically at $10 \mathrm{~s}$ intervals using a 160 computer. Five repeat measurements were made.

161 The fraction of the non-functional active ingredient (sodium chloride) dissolved, $Y$, after a time, $162 t$, was determined as follows:

$$
Y=\left(\frac{\chi-\chi_{o}}{\chi_{\infty}-\chi_{o}}\right) \times 100 \%
$$

164 where $\chi$ is the conductivity of the solution at a time $t$, and $\chi_{o}$ and $\chi_{\infty}$ are the initial and final 165 conductivities $(\mu \mathrm{S} / \mathrm{cm})$. The Weibull distribution function was used to describe the data $[9,40]$. 


$$
Y=1-\exp \left(-\left(\frac{t-t_{0}}{\tau_{d}}\right)^{\xi}\right)
$$

170 functional active ingredient in each tablet, $m_{a}(\mathrm{mg})$, was determined from:

$$
m_{a}=\Delta \chi \lambda V_{s}=\left(\chi_{\infty}-\chi_{o}\right) \lambda V_{s}
$$

172 where $\lambda$ is a constant obtained from a calibration curve of the amount of $\mathrm{NaCl}$ as a function of $\Delta \chi$,

173 which is the change in conductivity of the solution caused by presence of a known mass active of

174 ingredient, and $V_{s}$ is the volume of the dissolution medium (ml). The mean of 10 measurements

175 was determined for each fill factor and the coefficient of variation of the non-functional active

176 ingredient in the tablets was determined using:

$$
n_{t}=\frac{\bar{\sigma}}{\bar{m}_{a}} \times 100 \%
$$

179 where $\bar{m}_{a}$ is the mean value of active ingredient composition in the tablets and $\bar{\sigma}$ is the standard

180 deviation of the non-functional active ingredient compositions.

\subsubsection{Determination of acceptance values}

182 The European Pharmacopea recommends assessing the content uniformity of tablets by

183 computing Acceptance Values (AV) from the concentrations of the active ingredient and their

184 standard deviations and comparing them with previously established ranges [41]. The AV is 185 calculated from:

$$
A V=|M-X|+k \bar{\sigma}
$$


187 where $M$ is the reference value, $X$ is the average value for individual tablets, $k$ is a constant equal 188 to 2.4 for $n=10$ ( $n=$ number of repeat measurements) and $\bar{\sigma}$ is the standard deviation. The content 189 of uniformity requirement is assumed to be met if the AV of the first set of 10 tablets is $\leq 15$. The 190 acceptance values of the tablet from the different batches are reported in Table 1. According to this 191 table the granulation batches with fill factors of only 0.31 and 0.34 would pass acceptance.

\section{3. Results}

\section{$193 \quad 3.1 \quad$ Bulk density}

194 Before compression of the granules into tablets, the bulk densities of the dried granules were

195 determined as outlined previously. Fig. 1 shows that there is a reduction in the bulk density of the 196 granules as the fill factor is increased. This can be attributed to the changes in the degree of 197 consolidation and compaction of the granules when the batch size is changed whilst maintaining the 198 other granulation conditions.

\section{$199 \quad 3.2 \quad$ Compression data}

$200 \quad$ Fig. 2 (a) shows the loading and unloading curves for the fill factors investigated and Fig. 2 (b)

201 shows the same data expressed as the pressure as a function of the strain, which was calculated from $202 \Delta / h_{0}$ where $h_{0}$ is the initial height of the granular bed and $\Delta$ is displacement. It is clear from Fig. 2 203 (b) that the maximum strain increases (54 to 59\%) as the fill factor decreases.

204 The increase in strain required to achieve a given compression force as the fill factor 205 decreases (Fig. 2b) is consistent with data published previously that showed an increase in the 206 strength, Young's modulus and yield stress with increasing fill factor [1]. This is exemplified in Fig. 2073 for the strength, which shows that the strength of the granules approximately doubles for the range 208 of fill factors examined. 
Fig. 4 shows that efficacy coefficient decreases as the fill factor increases with the values

210 being less than the lower ideal limit of $0.1 \%$ for the three largest fill factors. The trend is consistent

211 with the increase in granule strength since the propensity of granules to deform is important in the 212 development of a cohesive tablet.

\section{$213 \quad 3.3 \quad$ Mechanical properties of the tablets}

214 Results in Fig. 5 (a) shows that the tablet strength is reduced by $\sim 25 \%$ when the fill factor is

215 increased from 0.31 to 0.42 . Since the tablets were formed by the compression of granules of the

216 same mass, maximum pressure and compression speed, the differences in the tablet strength cannot

217 be attributed to the tabletting conditions. Consequently, they must arise from the differences in the

218 mechanical properties of the granules as exemplified in Fig. 3 and the trend is reflected in the

219 reduction of the efficacy coefficient. Fig. 5(b) also shows that there is a clear correlation between

220 the tablet strength and that of the granules. Moreover, the reduction of the tensile strength of the

221 tablets corresponds to a similar reduction of $\sim 30 \%$ in the specific fracture energy (Fig. 6).

\section{$222 \quad 3.4 \quad$ Effect of fill factor on tablet dissolution}

223 Since it has been shown that the fill factor or size of the batch affects the strength of the tablets

224 it is reasonable to expect that they should also have different dissolution rates and this is evident

225 from the data Fig. 7 (a). The symbols show the measured data points (an average of 5

226 measurements) and the error bars are the standard deviation. The continuous line through the data

227 points are fits to Eq. (11). The dissolution profiles shift to the left with increasing fill factor,

228 implying an increase in the dissolution rate. The parameter $\tau_{\mathrm{d}}$; which is the length of time it takes to

229 release $63.2 \%$ of the drug was obtained from non-linear regression of Eq. (11) to the dissolution 230 data. 
In our previous work similar procedure was done using granules in same size range to those

232 used for tableting in current study to obtain dissolution characteristics of the granules [3]. The

233 granule dissolution tests were performed using granules of the same mass as the tablets (100 $\mathrm{mg})$.

234 The results that were obtained showed that the dissolution time, $\tau_{\mathrm{g}}$ of the granules increased with 235 increasing fill factor $(\sim 4$ to $\sim 12 s)$. The correlation between the dissolution time of the tablets and

236 that of granules is shown in Fig 7 (b). This result is consistent with the decrease in tablet strength

237 with increasing fill factor since it is generally the case that there is a correlation of the rate

238 dissolution and the tablet strength [1]. The correlation between the mean tablet dissolution time and

239 the tablet strength is shown in Fig. 8. The data demonstrate that stronger tablets require a longer

240 time to dissolve compared to those that are weaker. On the other hand, there is a minimum strength

241 is required for packing and handling purposes, therefore a trade-off has to be made in producing

242 tablets sufficient strength to survive handling processes without compromising the dissolution

243 kinetics.

\section{$244 \quad 3.5 \quad$ Effect of batch size on tablet drug homogeneity}

245 The relative standard deviation of the non-functional active ingredient composition in different

246 tablets produced from granules made with different fill factors is presented in Fig. 9 (a). The

247 coefficient of variation of the tablet non-functional active ingredient increases with the batch size,

248 which would result in a similar variation in the active pharmaceutical ingredient (API) composition

249 for a real pharmaceutical tablet. A similar trend has been found for the dissolution characteristics of

250 granules [1]. In our previous work [1] the coefficient of variation of the non-functional active

251 ingredient of samples of granules $\left(\eta_{\mathrm{g}}\right)$ obtained from different fill factors was determined using the

252 same procedure described in section 2.5. The coefficient of variation of non-functional ingredient in

253 the granules data from [3] was then plotted Fig. 9 (b). Please note that the masses of granules used 
254 in these measurements were the same as tablet masses used in the current study. The results show

255 that there is a linear correlation of the coefficient of variations of the tablets and corresponding

256 granules. This is an interesting point to note since it implies that information about the content

257 homogeneity of the tablets can be inferred from tests performed on the granules even before the 258 tablets are produced.

\section{4. Discussion}

260 In the current work it was found that increasing the granulator fill factor results in an

261 increase in the strength of the granules and a decrease in their degree of compression. It has also 262 been observed previously that the compressibility of granules decreased with their strength [42].

263 Similarly it was reported that the degree of compression of microcrystalline cellulose pellets 264 decreased with increasing values of their crushing strength [32]. Recent work by Chan et al. [43] 265 showed that increasing the bed load (which is equivalent to increasing fill factor) results in an 266 increase in granule-blade bed stress and the effect was more pronounced at high impeller speeds. 267 The granules from larger batches are then more likely to be more consolidated than those from 268 smaller batches. Such strong granules would be less compressible compared to those that are weaker 269 as observed in the current work. Thus it may be concluded that the ability of granules to convert net 270 compaction energy to cohesion decreases with increasing fill factor. This is consistent with the 271 tablet strength data, which showed a reduction with increasing fill factor. 


\section{5. Conclusion}

The granulator fill factor has a profound effect on the compaction properties of the granules.

276 Those produced from smaller batch sizes have superior compaction properties than those from

277 larger batches. The degree of compression of the granules decreases with increasing fill factor. This

278 may be due structural changes in the granules as a result of the different batch sizes. Further work is

279 recommended to analyse the changes in the internal and surface properties of the granules. An

280 important novel finding of the current work is that the variation of the non-functional active

281 ingredient in tablets are significantly affected by the value of the fill factor.

\section{Reference}

283 [1] C. Mangwandi, M.J. Adams, M.J. Hounslow, A.D. Salman, Effect of batch size on mechanical 284 properties of granules in high shear granulation, Powder Technology, 206 (2011) 44-52. [2] C. Mangwandi, A.B. Albadarin, A.a.H. Al-Muhtaseb, S.J. Allen, G.M. Walker, Optimisation of high shear granulation of multicomponent fertiliser using response surface methodology, Powder 287 Technology, 238 (2013) 142-150.

288 [3] C. Mangwandi, L. JiangTao, A.B. Albadarin, S.J. Allen, G.M. Walker, Alternative method for producing organic fertiliser from anaerobic digestion liquor and limestone powder: High Shear wet granulation, Powder Technology, 233 (2013) 245-254.

291 [4] C. Mangwandi, L. JiangTao, A.B. Albadarin, S.J. Allen, G.M. Walker, The variability in 292 nutrient composition of Anaerobic Digestate granules produced from high shear granulation, Waste 293 Management, 33 (2013) 33-42.

294 [5] S.M. Iveson, S. Holt, S. Biggs, Advancing contact angle of iron ores as a function of their 295 hematite and goethite content: implications for pelletising and sintering, International Journal of 296 Mineral Processing, 74 (2004) 281-287.

297 [6] I. Quaicoe, A. Nosrati, W. Skinner, J. Addai-Mensah, Agglomeration behaviour and product 298 structure of clay and oxide minerals, Chemical Engineering Science, 98 (2013) 40-50.

299 [7] D. Ameye, E. Keleb, C. Vervaet, J.P. Remon, E. Adams, D.L. Massart, Scaling-up of a lactose 300 wet granulation process in Mi-Pro high shear mixers, European Journal of Pharmaceutical Sciences, $301 \quad 17$ (2002) 247-251.

302 [8] B. Campisi, D. Vojnovic, D. Chicco, R. Phan-Tan-Luu, Melt granulation in a high shear mixer: 303 optimization of mixture and process variables using a combined experimental design, Chemometrics 304 and Intelligent Laboratory Systems, 48 (1999) 59.

305 [9] A. Dévay, K. Mayer, S. Pál, I. Antal, Investigation on drug dissolution and particle 306 characteristics of pellets related to manufacturing process variables of high-shear granulation, 307 Journal of Biochemical and Biophysical Methods, 69 (2006) 197-205. 
[10] M. Landin, P. York, M.J. Cliff, R.C. Rowe, A.J. Wigmore, The effect of batch size on scale-up of a pharmaceutical granulation in a fixed bowl mixer granulator, International Journal of 310 Pharmaceutics, 134 (1996) 243-246.

311 [11] C. Mangwandi, M.J. Adams, M.J. Hounslow, A.D. Salman, An investigation of the influence 312 of process and formulation variables on mechanical properties of high shear granules using design of experiment, International Journal of Pharmaceutics, 427 (2012) 328-336. [12] P.W.S. Heng, L.W. Chan, L. zhu, Effects of process variables and their interactions on melt pelletization in a high shear mixer S.T.P. Pharma Science, 10 (2000) 165-172.

[13] T. Schaefer, B. Taagegaard, L.J. Thomsen, H. Gjelstrup Kristensen, Melt pelletization in a high shear mixer. IV. Effects of process variables in a laboratory scale mixer, European Journal of Pharmaceutical Sciences, 1 (1993) 125.

[14] P.G. Smith, A.W. Nienow, Particle growth mechanisms in fluidised bed granulation--I : The effect of process variables, Chemical Engineering Science, 38 (1983) 1223.

[15] C. Mangwandi, M.J. Adams, M.J. Hounslow, A.D. Salman, Effect of impeller speed on mechanical and dissolution properties of high-shear granules, Chemical Engineering Journal, 164 (2010) 305-315.

[16] C.J. Broadbent, J. Bridgwater, D.J. Parker, S.T. Keningley, P. Knight, A phenomenological study of a batch mixer using a positron camera, Powder Technology, 76 (1993) 317.

[17] A. Darelius, H. Brage, A. Rasmuson, I. Niklasson Björn, S. Folestad, A volume-based multidimensional population balance approach for modelling high shear granulation, Chemical Engineering Science, 61 (2006) 2482-2493. [18] P.R. Mort, Scale-up of binder agglomeration processes, Powder Technology, 150 (2005) 86.

[19] P.R. Mort, Scale-up and control of binder agglomeration processes -- Flow and stress fields, Powder Technology, 189 (2009) 313-317.

[20] T.K. Bock, U. Kraas, Experience with the Diosna mini-granulator and assessment of process scalability, European Journal of Pharmaceutics and Biopharmaceutics, 52 (2001) 297-303.

[21] A. Hassanpour, C.C. Kwan, B.H. Ng, N. Rahmanian, Y.L. Ding, S.J. Antony, X.D. Jia, M. Ghadiri, Effect of granulation scale-up on the strength of granules, Powder Technology, 189 (2009) 304-312.

[22] N. Rahmanian, M. Ghadiri, Y. Ding, Effect of scale of operation on granule strength in high shear granulators, Chemical Engineering Science, 63 (2008) 915.

[23] M. Landin, P. York, M.J. Cliff, R.C. Rowe, A.J. Wigmore, Scale-up of a pharmaceutical granulation in fixed bowl mixer-granulators, International Journal of Pharmaceutics, 133 (1996) $127-131$.

[24] K. Tereshita, T. Nishimura, , S. Natsuyama, Optimization of Operating Conditions in a HighShear Mixer Using DEM Model: Determination of Optimal Fill Level, Chem. Pharm. Bull., 50 (2002) 1550-1557.

[25] R. Thies, P. Kleinebudde, Melt pelletisation of a hygroscopic drug in a high shear mixer: Part 1. Influence of process variables, International Journal of Pharmaceutics, 188 (1999) 131. [26] J. Cruz, M. Blanco, Content uniformity studies in tablets by NIR-CI, Journal of Pharmaceutical and Biomedical Analysis, 56 (2011) 408-412.

352 [28] J.J. Moes, M.M. Ruijken, E. Gout, H.W. Frijlink, M.I. Ugwoke, Application of process 353 analytical technology in tablet process development using NIR spectroscopy: Blend uniformity, 
content uniformity and coating thickness measurements, International Journal of Pharmaceutics, 357 355 (2008) 108-118.

356 [29] B. Vajna, I. Farkas, A. Szabo, Z. Zsigmond, G. Marosi, Raman microscopic evaluation of technology dependent structural differences in tablets containing imipramine model drug, Journal of Pharmaceutical and Biomedical Analysis, 51 (2010) 30-38. [30] Z. Wu, O. Tao, W. Cheng, L. Yu, X. Shi, Y. Qiao, Visualizing excipient composition and homogeneity of Compound Liquorice Tablets by near-infrared chemical imaging, Spectrochimica Acta Part A: Molecular and Biomolecular Spectroscopy, 86 (2012) 631-636.

[31] M.J. Adams, M.A. Mullier, J.P.K. Seville, Agglomerate strength measurement using a uniaxial confined compression test, Powder Technology, 78 (1994) 5.

[32] B. Johansson, M. Wikberg, R. Ek, G. Alderborn, Compression behaviour and compactability of microcrystalline cellulose pellets in relationship to their pore structure and mechanical properties, International Journal of Pharmaceutics, 117 (1995) 57.

[33] C. Mangwandi, A.B. Albadarin, L. JiangTao, S. Allen, G.M. Walker, Development of a valueadded soil conditioner from high shear co-granulation of organic waste and limestone powder, Powder Technology, 252 (2014) 33-41.

[34] J.T. Fell, J.M. Newton, Determination of tablet strength by the diametral-compression test, Journal of Pharmaceutical Sciences, 59 (1970) 688-691.

[35] F. Chantraine, M. Viana, S. Cazalbou, N. Brielles, O. Mondain-Monval, C. Pouget, P. Branlard, G. Rubinstenn, D. Chulia, From compressibility to structural investigation of sodium dodecyl sulphate -- Part 2: A singular behavior under pressure, Powder Technology, 177 (2007) 41. [36] F. Chantraine, M. Viana, S. Cazalbou, N. Brielles, O. Mondain-Monval, C. Pouget, P. Branlard, G. Rubinstenn, D. Chulia, From compressibility to structural investigation of sodium dodecyl sulphate -- Part 1: Powder and tablet physico-chemical characteristics, Powder Technology, 177 (2007) 34.

[37] F. Chantraine, M. Viana, S. Cazalbou, N. Brielles, O. Mondain-Monval, C. Pouget, P. Branlard, G. Rubinstenn, D. Chulia, From compressibility to structural investigation of sodium dodecyl sulphate -- Part 2: A singular behavior under pressure, Powder Technology, 177 (2007) 4150.

[38] C. Pontier, E. Champion, M. Viana, D. Chulia, D. Bernache-Assollant, Use of cycles of compression to characterize the behaviour of apatitic phosphate powders, Journal of the European Ceramic Society, 22 (2002) 1205.

[39] M. Viana, J. Ribet, F. Rodriguez, D. Chulia, Powder functionality test: a methodology for rheological and mechanical characterization, Pharmaceutical development and technology, 10 (2005) 327.

[40] F. Langenbucher, Parametric representation of dissolution-rate curves by the RRSBW distribution, Pharm Ind 38 (1976) 472-477.

[41] E.P. 2008, Uniformity of Dosage Units, European Council2008, pp. 327-330.

[42] H. Santos, F. Veiga, M.E. Pina, J.J. Sousa, Compaction, compression and drug release characteristics of xanthan gum pellets of different compositions, European Journal of Pharmaceutical Sciences, 21 (2004) 271.

[43] E.L. Chan, G.K. Reynolds, B. Gururajan, M.J. Hounslow, A.D. Salman, Blade-granule bed stress in a cylindrical high shear granulator: I-Online measurement and characterisation, Chemical Engineering Science, 86 (2013) 38-49. 


\section{List of Tables.}

Table 1: Summary of non-functional active ingredient composition, acceptance values for tablets from different batch sizes

\section{List of figures}

Fig. 1: Bulk density of the granules in the size range $0.5-0.6 \mathrm{~mm}$.

Fig. 2: a) Force-displacement profiles for the four different fill factors and (b) applied bed pressure as function of bed strain.

Fig. 3: Effect of fill factor on granule strength parameter.

Fig. 4: Efficacy coefficient as a function of fill factor.

Fig. 5: (a) Tablet tensile strength as a function of fill factor and (b) correlation between granule and tablet strength.

Fig. 6: Specific fracture energy of the tablets as a function of the fill factor

Fig. 7: (a) Tablet dissolution profiles for fill factor and (b) dissolution time of the tablets vs dissolution time for granules.

Fig. 8: Correlation between the mean dissolution time and the strength of the tablets.

Fig. 9: (a) Coefficient of variation of the tablet non-functional active ingredient content as a function of fill factor and (b) correlation between $\eta_{t}$ and $\eta_{g}$. 
426 Table 1: Summary of non-functional active ingredient composition, acceptance values for tablets from 427 different batch sizes.

\begin{tabular}{ccccc}
\hline & Average & & & \\
& composition & Reference & Percentage & Acceptance \\
& $\bar{m}_{a i, \text { tab }}$ & composition & Average & value \\
Fill factor & $(\mathrm{mg})$ & $(\%)$ & composition $\mathrm{fX}$ & AV \\
$(-)$ & 1.83 & 100 & 91.6 & $(-)$ \\
\hline 0.31 & 1.88 & 100 & 94.0 & 8.7 \\
0.34 & 1.59 & 100 & 79.5 & 6.4 \\
0.38 & 1.78 & 100 & 89.0 & 21.1 \\
0.41 & & & & 11.7 \\
\hline
\end{tabular}




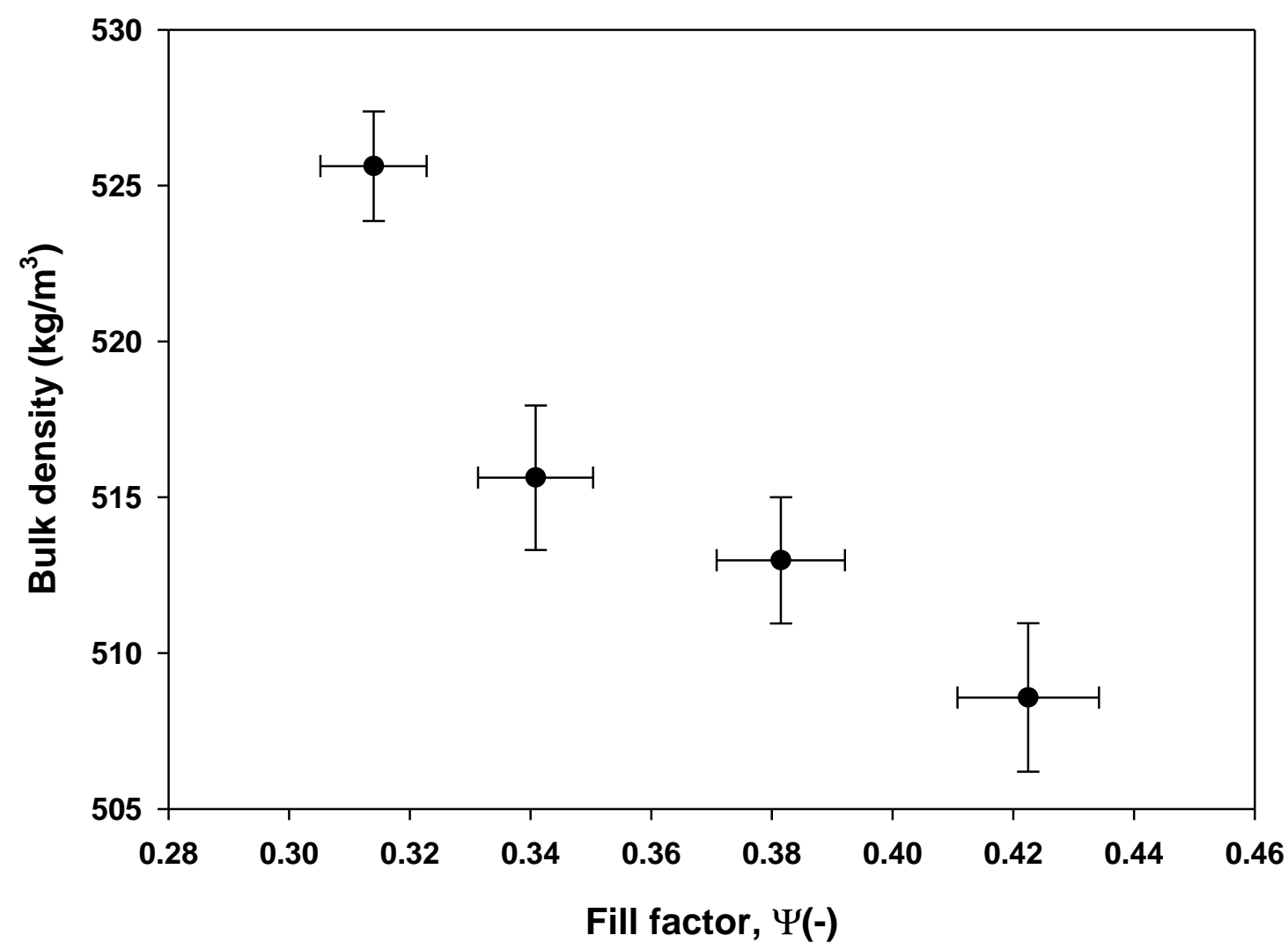

431

Fig. 1: Bulk density of the granules in the size range $0.5-0.6 \mathrm{~mm}$.

433 


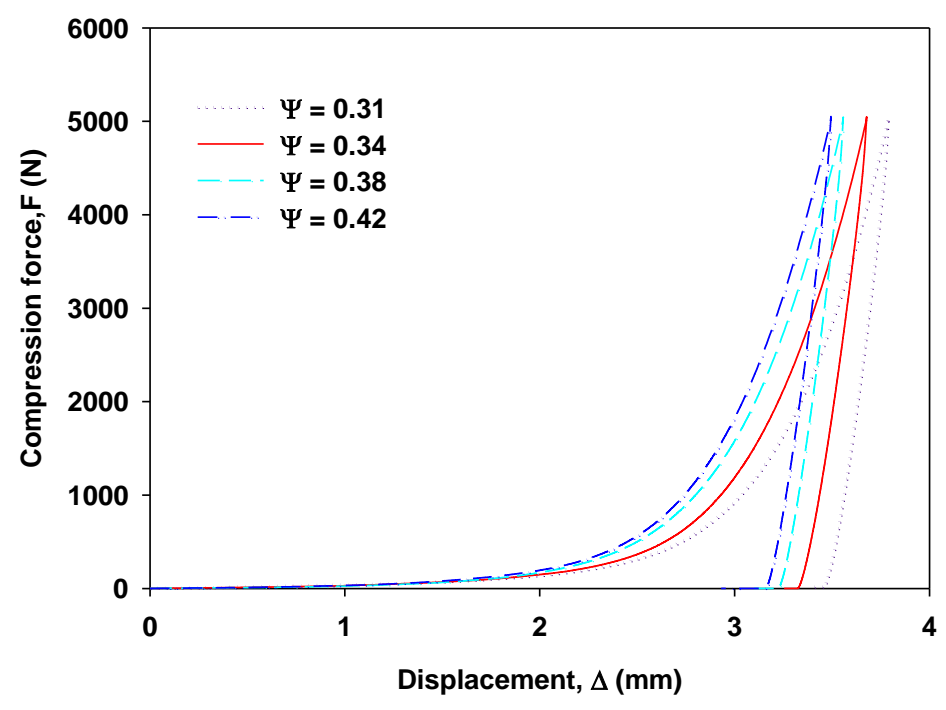

(a)

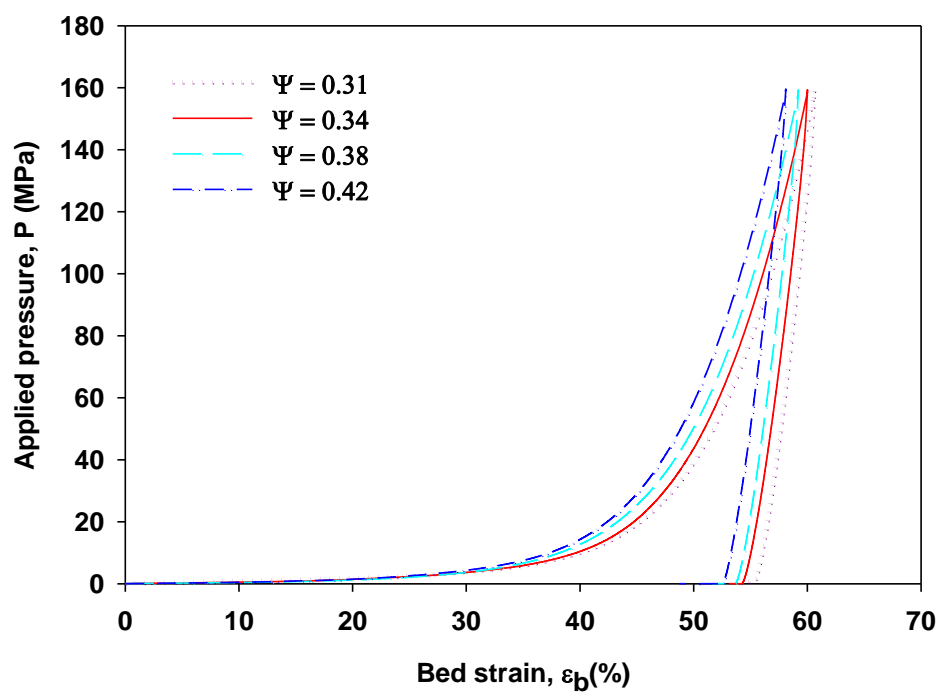

(b)

435 Fig. 2: a) Force-displacement profiles for the four different fill factors and (b) applied bed pressure as 436 function of bed strain. 


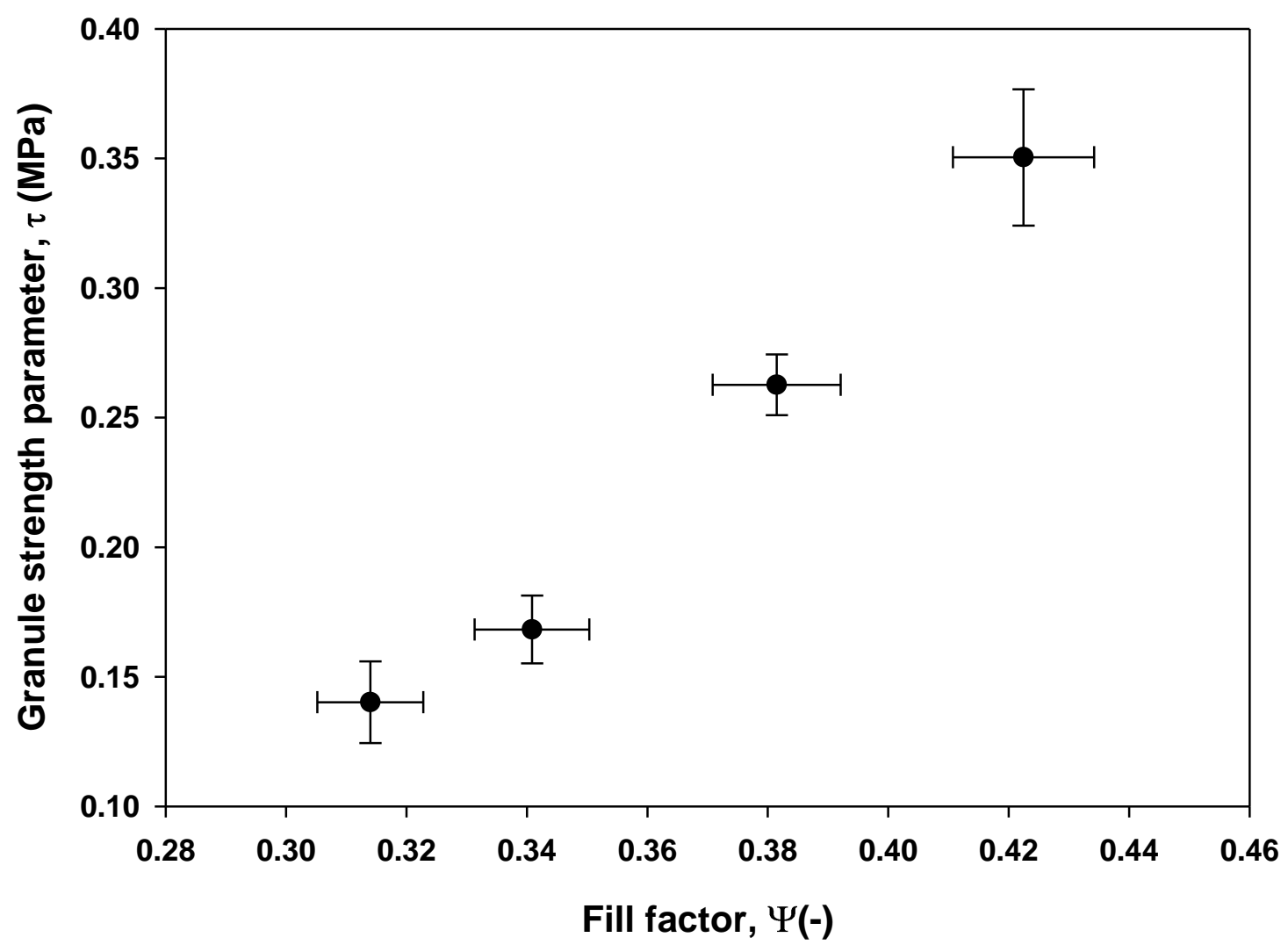

439

$440 \quad$ Fig. 3: Effect of fill factor on granule strength parameter.

441 


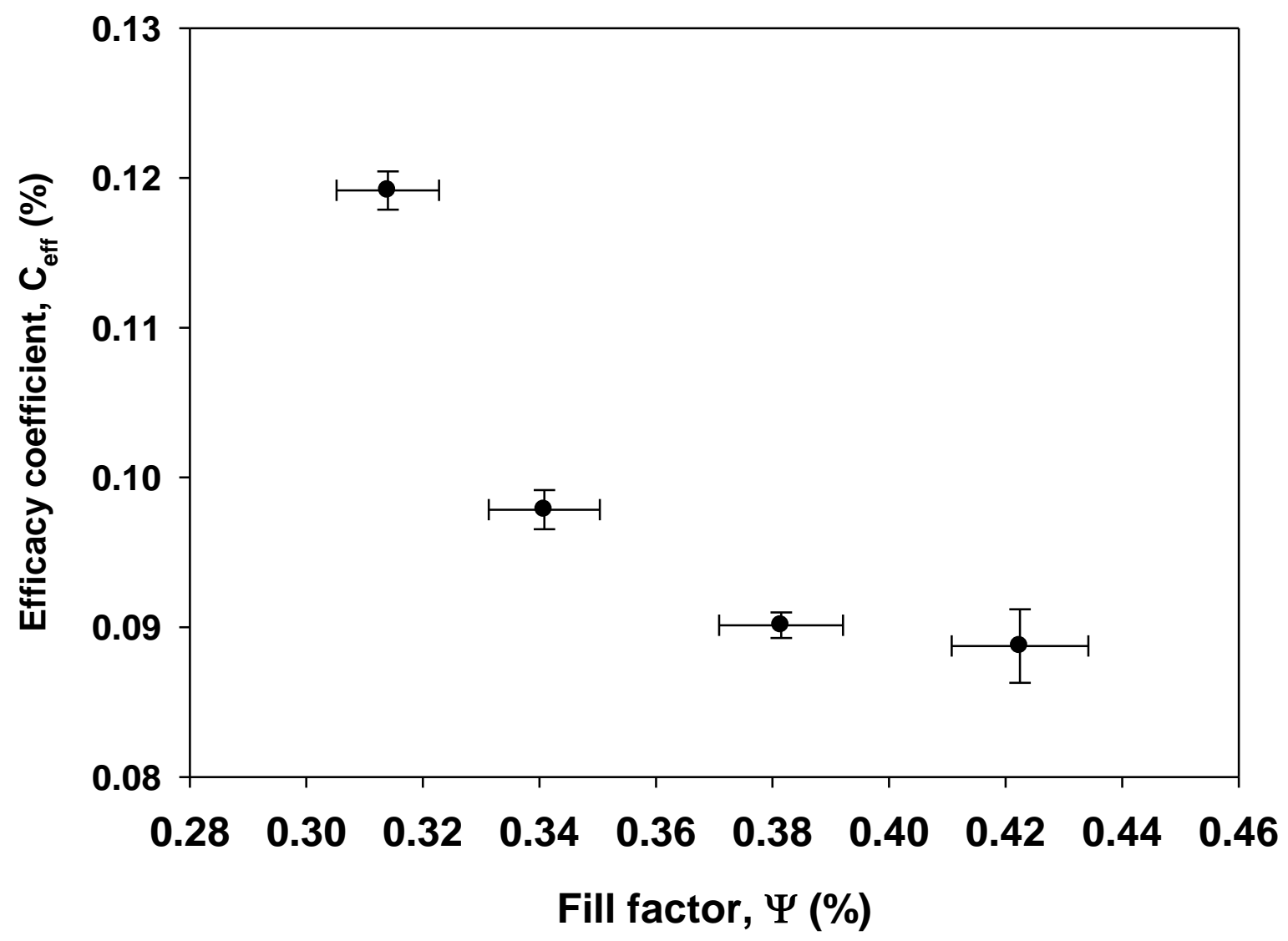

443

$444 \quad$ Fig. 4: Efficacy coefficient as a function of fill factor.

445 


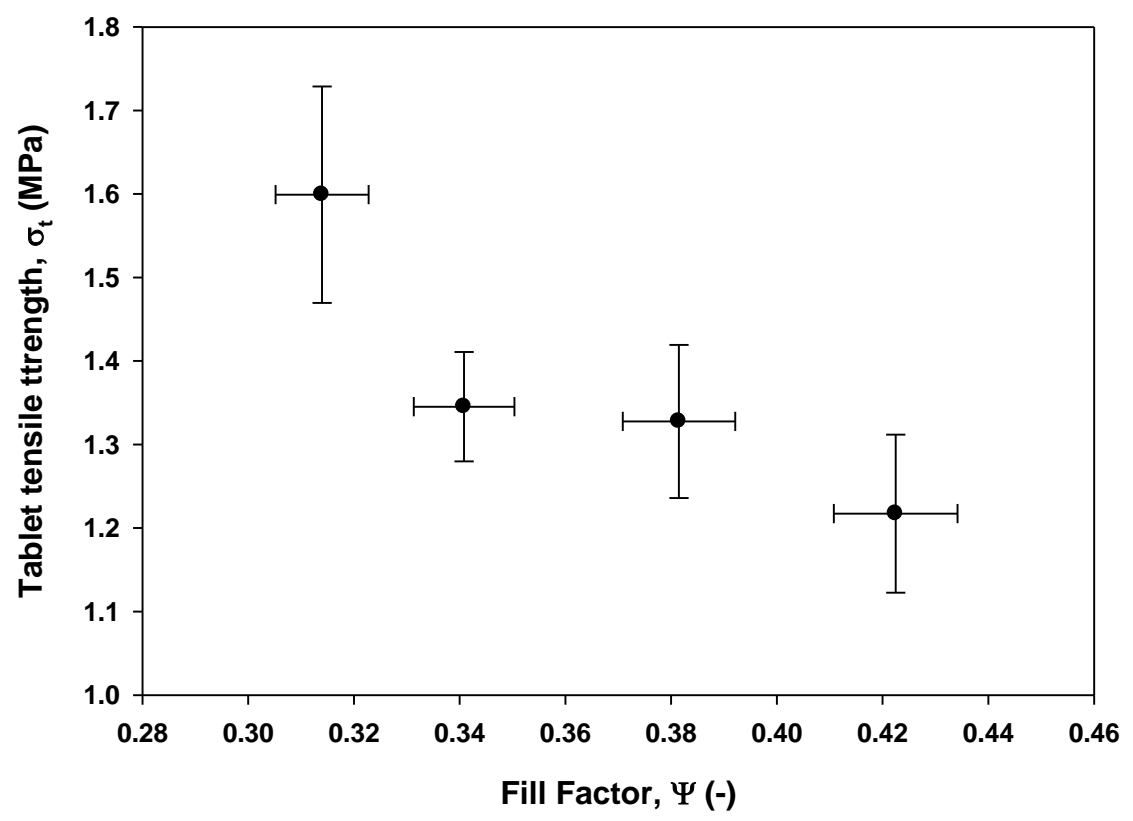

(a)

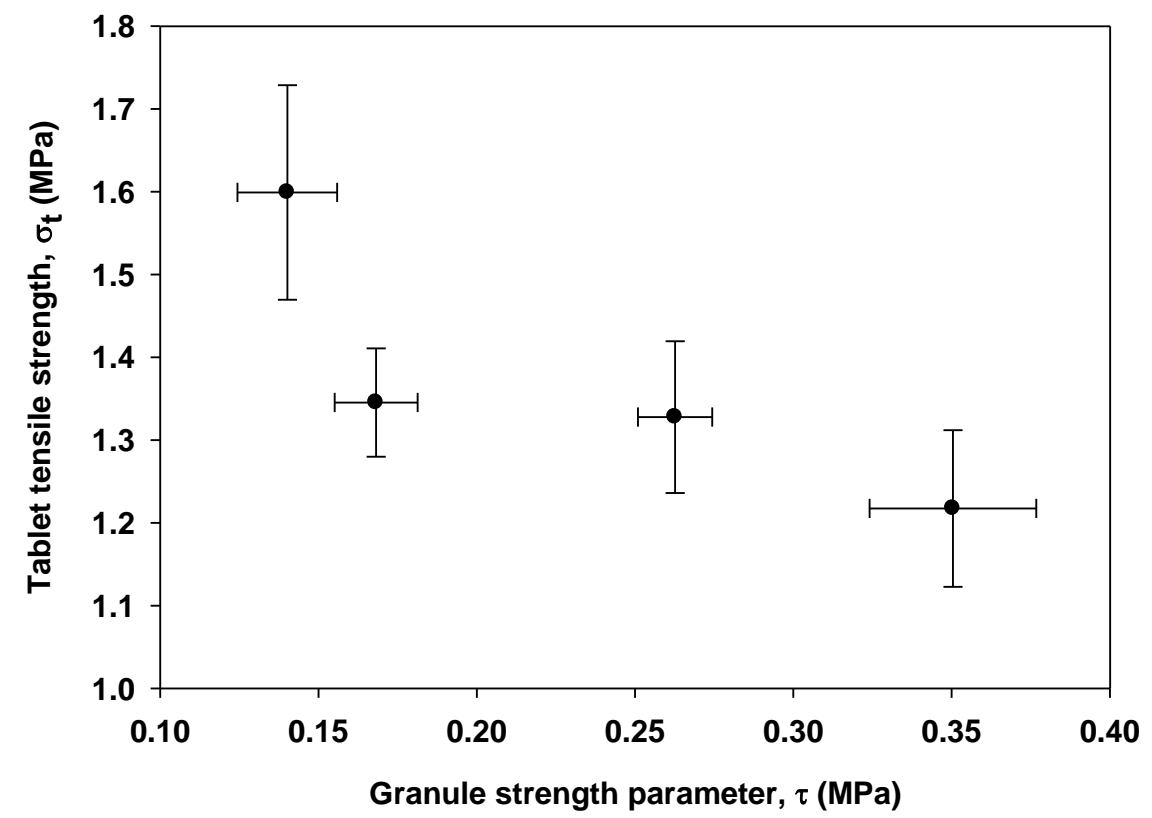

(b)

448 Fig. 5: (a) Tablet tensile strength as a function of fill factor and (b) correlation between granule and tablet 449 strength. 


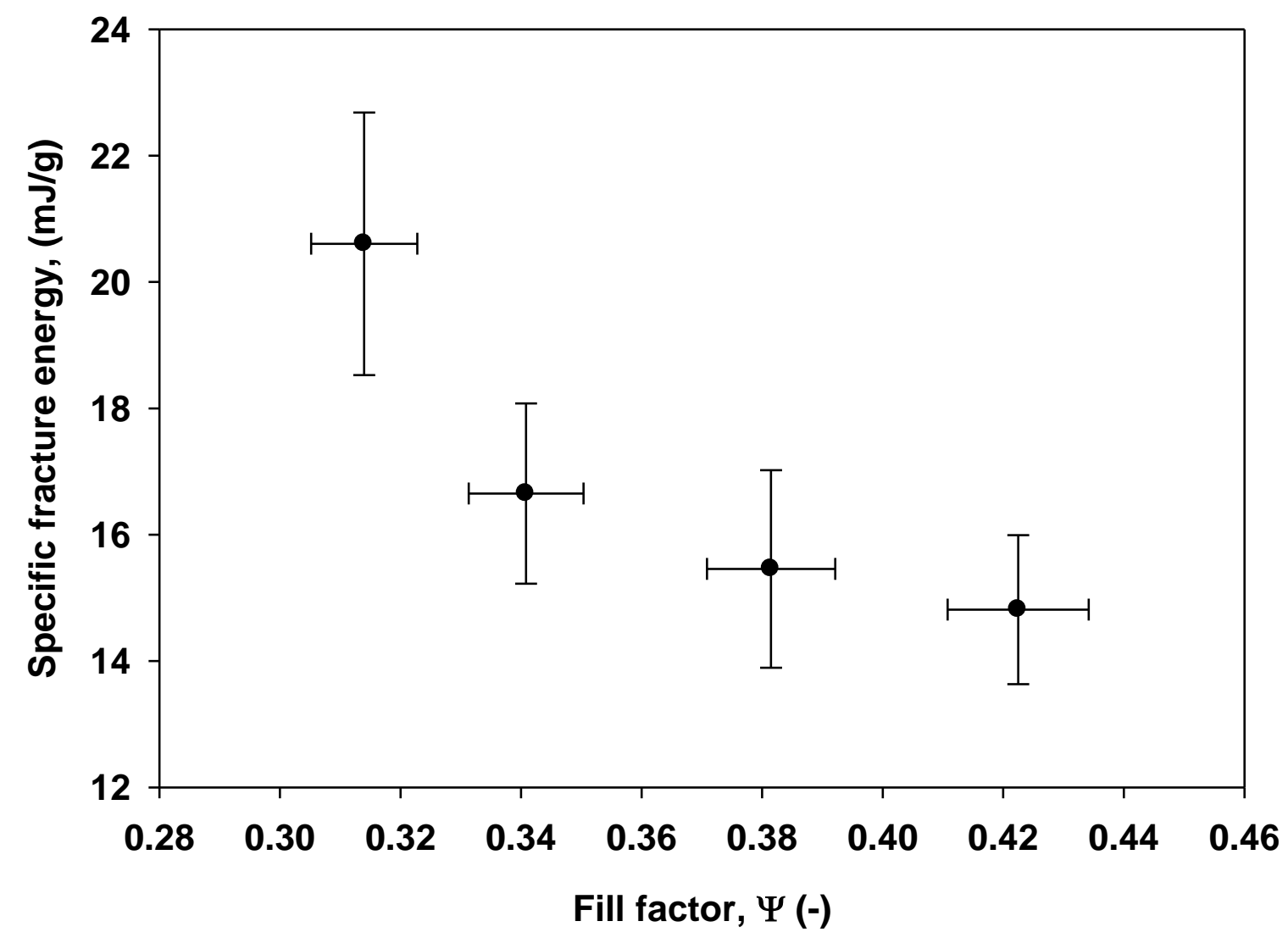

452 Fig. 6: Specific fracture energy of the tablets as a function of the fill factor 453 


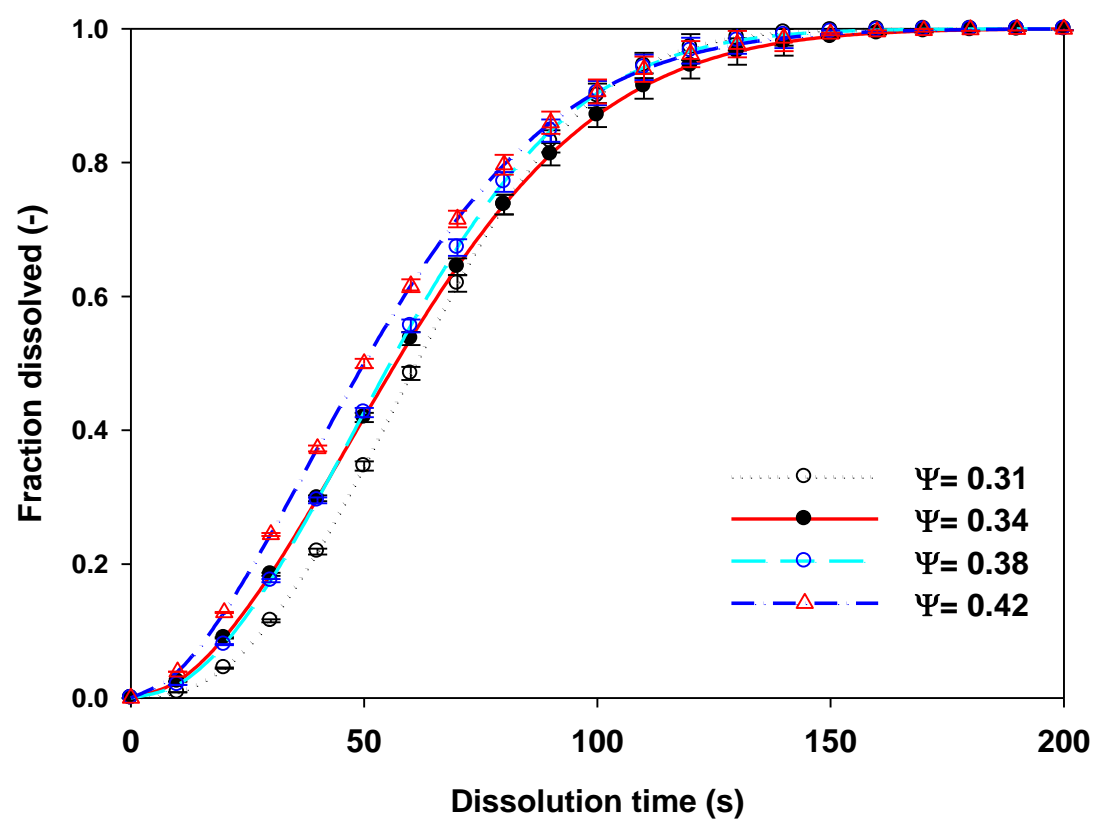

(a)

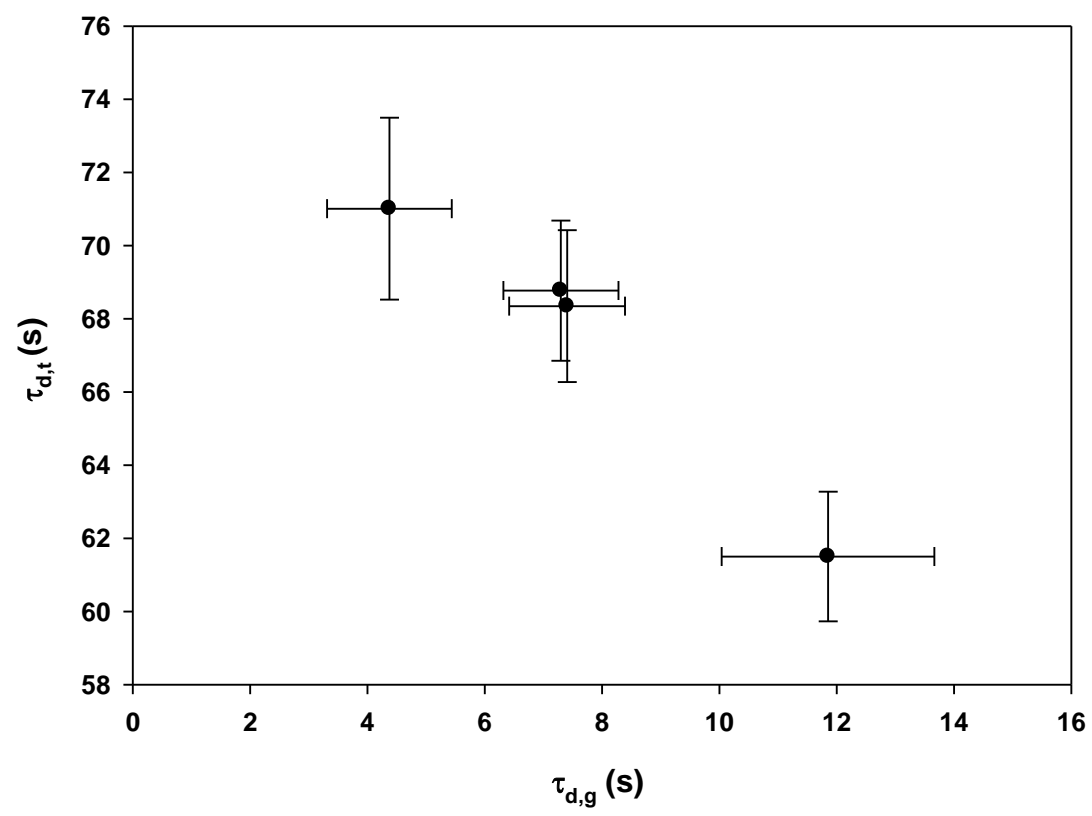

(b)

455 Fig. 7: (a) Tablet dissolution profiles for fill factor and (b) dissolution time of the tablets vs dissolution time 456 for granules. 


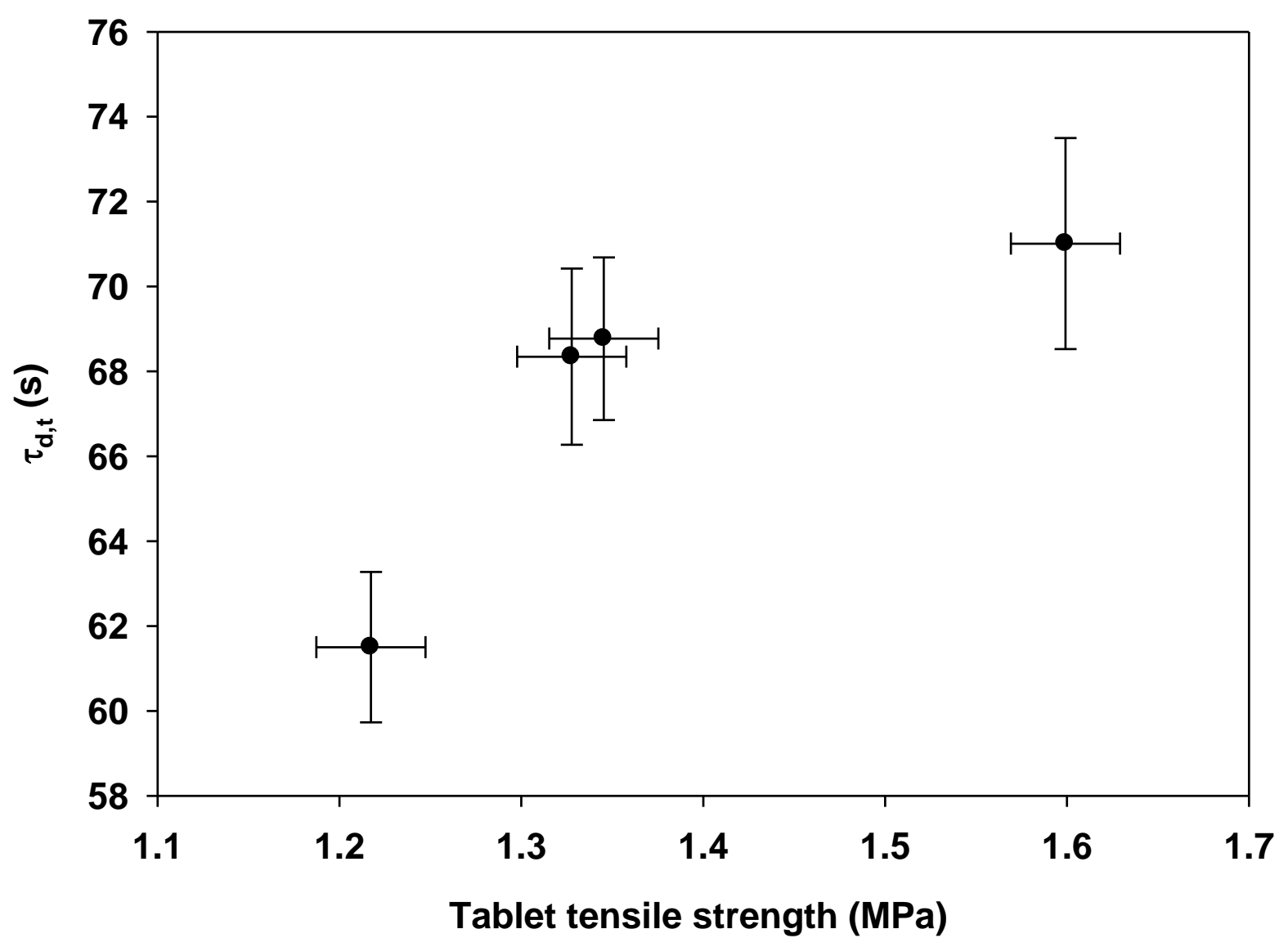

459 Fig. 8: Correlation between the mean dissolution time and the strength of the tablets.

460 


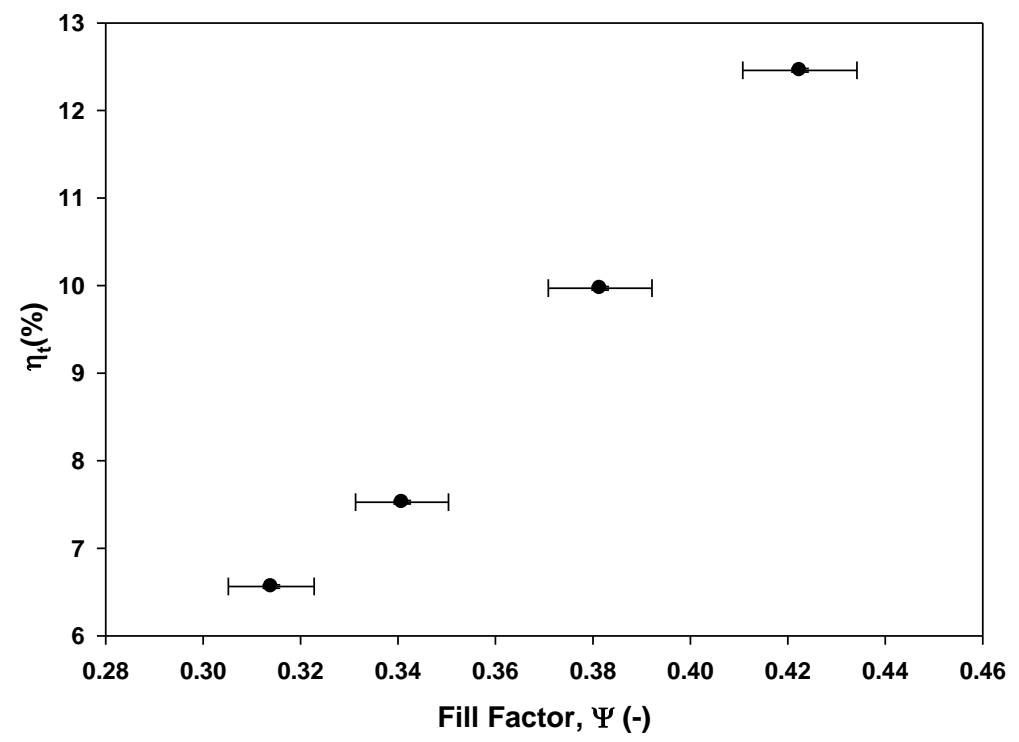

(a)

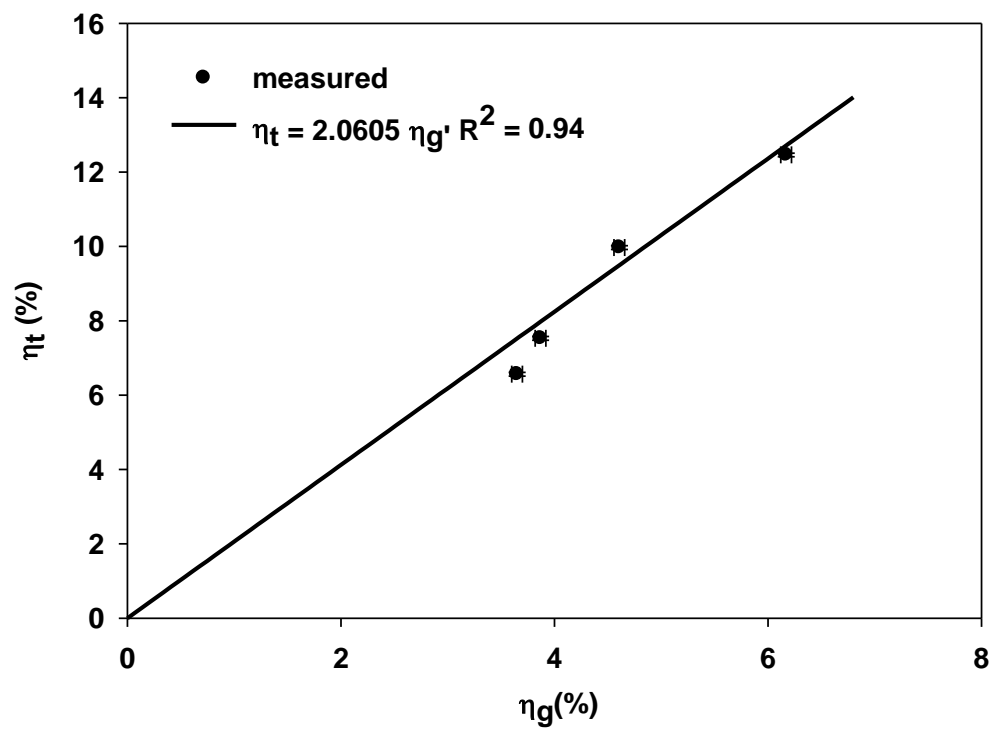

(b)

461

462

463

464

465

466
Fig. 9: (a) Coefficient of variation of the tablet non-functional active ingredient content as a function of fill factor and (b) correlation between $\eta_{\mathrm{t}}$ and $\eta_{\mathrm{g}}$. 